\title{
Optomechanically induced amplification and perfect transparency in double-cavity optomechanics
}

\author{
Xiao-Bo Yan $^{1,2, *}$, W. Z. Jia ${ }^{3}$, Yong $\mathbf{L i}^{4}$, Jin-Hui $\mathbf{W u}^{2, \dagger}$, Xian-Li Li ${ }^{1}$, Hai-Wei $\mathbf{M u}^{1}$ \\ ${ }^{1}$ College of Electronic Science, Northeast Petroleum University, Daqing 163318, China \\ ${ }^{2}$ College of Physics, Jilin University, Changchun 130012, China \\ ${ }^{3}$ Quantum Optoelectronics Laboratory, School of Physical Science and Technology, \\ Southwest Jiaotong University, Chengdu 610031, China \\ ${ }^{4}$ Beijing Computational Science Research Center, Beijing 100084, China \\ Corresponding authors. E-mail: *xiaoboyan@126.com, ${ }^{\dagger} j h w u @ j l u . e d u . c n$ \\ Received October 18, 2014; accepted December 4, 2014
}

\begin{abstract}
We study optomechanically induced amplification and perfect transparency in a double-cavity optomechanical system. We find that if two control lasers with appropriate amplitudes and detunings are applied to drive the system, optomechanically induced amplification of a probe laser can occur. In addition, perfect optomechanically induced transparency, which is robust to mechanical dissipation, can be realized by the same type of driving. These results indicate important progress toward signal amplification, light storage, fast light, and slow light in quantum information processes.
\end{abstract}

Keywords optomechanics, optomechanically induced amplification, optomechanically induced transparency

PACS numbers 42.65.Yj, 03.65.Ta, 42.50.Wk

\section{Introduction}

Cavity optomechanics, which explores the interaction between light fields and mechanical motion, has attracted much attention in the past few years for its potential application in the ultrasensitive detection of tiny masses, forces, and displacements [1-5]. One standard optomechanical setup, and the simplest, is a Fabry-Perot cavity in which one end mirror is a micro- or nanomechanical vibrating object [6-8]. Various other optomechanical experimental systems have been designed and investigated, such as silica toroidal optical microresonators [911], photonic crystal cavities [12, 13], micromechanical membranes $[14,15]$, typical optomechanical cavities confining cold atoms [16-18], and superconducting circuits $[19,20]$.

Typically, when an optomechanical cavity is driven by a red-detuned laser, a mechanical oscillator can be cooled to its quantum ground state [21-23]. Moreover, in this red-detuned regime, some well-known phenomena in atomic ensembles can find their analog in optomechanical systems. Specifically, under strong driving, normal mode splitting [24-26] (called Autler-Townes effects in atomic physics) can be observed. In contrast, for rela- tively weak driving (much less than the cavity dissipation rate), an electromagnetically induced transparencylike phenomenon called optomechanically induced transparency has been theoretically predicted $[8,27]$ and experimentally verified $[26,28-30]$. This phenomenon can be used to slow and even stop light signals [31, 32] in long-lived mechanical vibrations. On the other hand, when a driving laser is applied to the mechanical blue sideband, the mechanical element of an optomechanical system can be heated, leading to phonon lasing [33-35] and probe amplification [29, 30, 36-38].

In our previous work [39], we investigated coherent perfect transmission and absorption in a double-cavity optomechanical system in which the mechanical resonator (MR) is totally reflective [23, 40-44], driven by two pump fields applied to the mechanical red sideband. In this paper, we study optomechanically induced amplification and perfect transparency in the same system under a different type of driving. We find that if the doublecavity optomechanical system is driven by a red sideband laser from one side and a blue sideband laser from the other side and the amplitudes of the lasers are manipulated appropriately, optomechanically induced amplification can occur for a nearly resonant weak signal field (probe field). In addition, by adjusting the control fields, 
an interesting perfect optomechanically induced transparency (with a transmission coefficient rigorously equal to 1) can be realized under the same type of driving. When this perfect transmission occurs, a quantum coherence process due to the double driving can totally suppress the decoherence due to the dissipation of the MR. This double-driving device could be used to realize optical signal amplifiers, switches, memory, and so on.

The rest of this paper is organized as follows. In Section 2, we introduce the double-cavity optomechanical model, obtain the equations of motion for the MR and the two cavity modes, solve these equations, and obtain the output fields. In Section 3, we show how to realize perfect optomechanically induced transparency, although with a large mechanical decay rate $\gamma_{m}$. In Section 4 , we show how to realize optomechanically induced amplification for a weak signal field (probe field) while keeping the system below the phonon lasing threshold. The conclusions are presented in Section 5.

\section{Model and equations}

We consider a double-cavity hybrid system with one perfectly reflective MR inserted between two fixed partially transmissive mirrors [23, 40-44] (see Fig. 1). It is worth emphasizing that the double-cavity model here is very different from that studied in Refs. [14] and [45], in which the MR is partially transmissive. The MR has an eigenfrequency $\omega_{m}$ and a decay rate $\gamma_{m}$ and thus has a mechanical quality factor $Q$ of $\omega_{m} / \gamma_{m}$. Two identical optical cavities of length $L$ and frequency $\omega_{0}$ are obtained when the $\mathrm{MR}$ is at its equilibrium position in the absence of external excitation. We describe the two optical modes by annihilation (creation) operators $c_{1}\left(c_{1}^{\dagger}\right)$ and $c_{2}\left(c_{2}^{\dagger}\right)$, and the only mechanical mode is denoted by $b\left(b^{\dagger}\right)$. These annihilation and creation operators are restricted by the commutation relation $\left[c_{i}, c_{i}^{\dagger}\right]=1(i=1,2),\left[c_{1}, c_{2}\right]=0$, and $\left[b, b^{\dagger}\right]=1$. Two coupling fields with amplitudes $\varepsilon_{c}=\sqrt{2 \kappa \wp_{c} /\left(\hbar \omega_{c}\right)}$ and $\varepsilon_{d}=\sqrt{2 \kappa \wp_{d} /\left(\hbar \omega_{d}\right)}$ are used to drive the double-cavity system from either the left or right fixed mirror, and one probe field is injected into the left optical cavity with an amplitude $\varepsilon_{p}=\sqrt{2 \kappa \wp_{p} /\left(\hbar \omega_{p}\right)}$. Here, $\wp_{c}, \wp_{d}$, and $\wp_{p}$ are the respective field powers, $\kappa$ is the common decay rate of both cavity modes, and $\omega_{c}, \omega_{d}$, and $\omega_{p}$ are the respective field frequencies. Then the total Hamiltonian in the rotating-wave frame of frequency $\omega_{c}+\omega_{d}$ can be written as

$$
\begin{aligned}
H= & \hbar \Delta_{c} c_{1}^{\dagger} c_{1}+\hbar \Delta_{d} c_{2}^{\dagger} c_{2}+\hbar g_{0}\left(c_{2}^{\dagger} c_{2}-c_{1}^{\dagger} c_{1}\right)\left(b^{\dagger}+b\right) \\
& +\hbar \omega_{m} b^{\dagger} b+\mathrm{i} \hbar \varepsilon_{c}\left(c_{1}^{\dagger}-c_{1}\right)+\mathrm{i} \hbar \varepsilon_{d}\left(c_{2}^{\dagger}-c_{2}\right) \\
& +\mathrm{i} \hbar\left(c_{1}^{\dagger} \varepsilon_{p} \mathrm{e}^{-\mathrm{i} \delta t}-c_{1} \varepsilon_{p}^{*} \mathrm{e}^{\mathrm{i} \delta t}\right)
\end{aligned}
$$

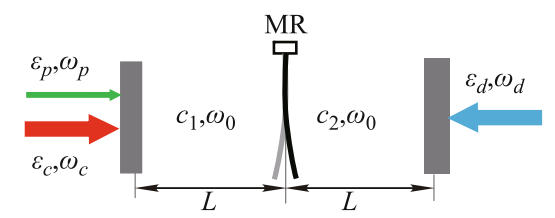

Fig. 1 A double-cavity optomechanical system with a mechanical resonator (MR) inserted between two fixed mirrors. The two cavities have identical cavity lengths $L$ and mode frequencies $\omega_{0}$ in the absence of radiation pressure. Coupling field and driving field with frequencies $\omega_{c}, \omega_{d}$ and amplitudes $\varepsilon_{c}, \varepsilon_{d}$, respectively, act upon opposite sides of the double-cavity system. The probe field with frequency $\omega_{p}$ and amplitude $\varepsilon_{p}$ is injected into the left optical cavity.

where $\Delta_{c}=\omega_{0}-\omega_{c}\left(\Delta_{d}=\omega_{0}-\omega_{d}\right)$ is the detuning between the cavity modes and coupling field (driving field), $\delta=\omega_{p}-\omega_{c}$ is the detuning between the probe field and the coupling field, and $g_{0}=\frac{\omega_{0}}{L} \sqrt{\frac{\hbar}{2 m \omega_{m}}}$ is the hybrid coupling constant between the mechanical and optical modes.

The dynamics of the system is described by the quantum Langevin equations for the relevant annihilation operators of the mechanical and optical modes,

$$
\begin{aligned}
& \dot{b}=-\mathrm{i} \omega_{m} b-\mathrm{i} g_{0}\left(c_{2}^{\dagger} c_{2}-c_{1}^{\dagger} c_{1}\right)-\frac{\gamma_{m}}{2} b+\sqrt{\gamma_{m}} b_{i n}, \\
& \dot{c}_{1}=-\left[\kappa+\mathrm{i} \Delta_{c}-\mathrm{i} g_{0}\left(b^{\dagger}+b\right)\right] c_{1}+\varepsilon_{c}+\varepsilon_{p} \mathrm{e}^{-\mathrm{i} \delta t}+\sqrt{2 \kappa} c_{1}^{i n}, \\
& \dot{c}_{2}=-\left[\kappa+\mathrm{i} \Delta_{d}+\mathrm{i} g_{0}\left(b^{\dagger}+b\right)\right] c_{2}+\varepsilon_{d}+\sqrt{2 \kappa} c_{2}^{i n},
\end{aligned}
$$

where $b_{i n}$ is the thermal noise on the MR with zero mean value, and $c_{1}^{i n}\left(c_{2}^{i n}\right)$ is the input quantum vacuum noise from the left (right) cavity with zero mean value. Because we consider the mean response of the system, we do not include these noise terms in the following discussions. In the absence of the probe field, Eq. (2) can be solved with the factorization assumption $\left\langle b c_{i}\right\rangle=\langle b\rangle\left\langle c_{i}\right\rangle$ to generate the steady-state mean values

$$
\begin{aligned}
& \langle b\rangle=b_{s}=\frac{-\mathrm{i} g_{0}\left(\left|c_{2 s}\right|^{2}-\left|c_{1 s}\right|^{2}\right)}{\frac{\gamma_{m}}{2}+\mathrm{i} \omega_{m}}, \\
& \left\langle c_{1}\right\rangle=c_{1 s}=\frac{\varepsilon_{c}}{\kappa+\mathrm{i} \Delta_{1}}, \\
& \left\langle c_{2}\right\rangle=c_{2 s}=\frac{\varepsilon_{d}}{\kappa+\mathrm{i} \Delta_{2}},
\end{aligned}
$$

where $\Delta_{1,2}=\Delta_{c, d} \mp g_{0}\left(b_{s}+b_{s}^{*}\right)$ denotes the effective detuning between the cavity modes and the coupling and driving fields when the membrane oscillator deviates from its equilibrium position. Note in particular that $g_{0}\left|b_{s}\right|$ is typically very small compared to $\omega_{m}$ and even becomes exactly zero for $\left|c_{1 s}\right|=\left|c_{2 s}\right|\left(\left|\varepsilon_{c}\right|=\left|\varepsilon_{d}\right|\right)$.

In the presence of a probe field, however, we can write each operator as the sum of its mean value and its small fluctuation $\left(b=b_{s}+\delta b, c_{1}=c_{1 s}+\delta c_{1}, c_{2}=c_{2 s}+\delta c_{2}\right)$ to solve Eq. (2) when the coupling field and driving field are 
sufficiently strong. Then, keeping only the linear terms of the fluctuation operators and moving into an interaction scenario by introducing $\delta b \rightarrow \delta b \mathrm{e}^{-\mathrm{i} \omega_{m} t}, \delta c_{1} \rightarrow \delta c_{1} \mathrm{e}^{-\mathrm{i} \Delta_{1} t}$, and $\delta c_{2} \rightarrow \delta c_{2} \mathrm{e}^{-\mathrm{i} \Delta_{2} t}$, we obtain the linearized quantum Langevin equations

$$
\begin{aligned}
\delta \dot{b}= & -\mathrm{i} g_{0}\left(c_{2 s}^{*} \delta c_{2} \mathrm{e}^{-\mathrm{i}\left(\Delta_{2}-\omega_{m}\right) t}-c_{1 s}^{*} \delta c_{1} \mathrm{e}^{-\mathrm{i}\left(\Delta_{1}-\omega_{m}\right) t}\right) \\
- & \mathrm{i} g_{0}\left(c_{2 s} \delta c_{2}^{\dagger} \mathrm{e}^{\mathrm{i}\left(\Delta_{2}+\omega_{m}\right) t}-c_{1 s} \delta c_{1}^{\dagger} \mathrm{e}^{\mathrm{i}\left(\Delta_{1}+\omega_{m}\right) t}\right)-\frac{\gamma_{m}}{2} \delta b, \\
\delta \dot{c}_{1}= & -\kappa \delta c_{1}+\mathrm{i} g_{0} c_{1 s}\left(\delta b \mathrm{e}^{-\mathrm{i}\left(\omega_{m}-\Delta_{1}\right) t}+\delta b^{\dagger} \mathrm{e}^{\mathrm{i}\left(\omega_{m}+\Delta_{1}\right) t}\right) \\
& +\varepsilon_{p} \mathrm{e}^{-\mathrm{i}\left(\delta-\Delta_{1}\right) t}, \\
\delta \dot{c}_{2}= & -\kappa \delta c_{2}-\mathrm{i} g_{0} c_{2 s}\left(\delta b \mathrm{e}^{-\mathrm{i}\left(\omega_{m}-\Delta_{2}\right) t}+\delta b^{\dagger} \mathrm{e}^{\mathrm{i}\left(\omega_{m}+\Delta_{2}\right) t}\right) .
\end{aligned}
$$

If the coupling field drives the mechanical red sideband while the driving field drives the blue sideband $\left(\Delta_{1} \approx \omega_{m}, \Delta_{2} \approx-\omega_{m}\right)$, the hybrid system is operating in the resolved sideband regime $\left(\omega_{m} \gg \kappa\right)$, the membrane oscillator has a high mechanical quality factor $\left(\omega_{m} \gg \gamma_{m}\right)$, and the mechanical frequency $\omega_{m}$ is much larger than $g_{0}\left|c_{1 s}\right|$ and $g_{0}\left|c_{2 s}\right|$, Eq. (4) will be simplified to

$$
\begin{aligned}
& \delta \dot{b}=-\mathrm{i} g_{0}\left(c_{2 s} \delta c_{2}^{\dagger}-c_{1 s}^{*} \delta c_{1}\right)-\frac{\gamma_{m}}{2} \delta b \\
& \delta \dot{c}_{1}=-\kappa \delta c_{1}+\mathrm{i} g_{0} c_{1 s} \delta b+\varepsilon_{p} \mathrm{e}^{-\mathrm{i} x t} \\
& \delta \dot{c}_{2}=-\kappa \delta c_{2}-\mathrm{i} g_{0} c_{2 s} \delta b^{\dagger}
\end{aligned}
$$

where $x=\delta-\omega_{m}$. We can examine the expectation values of small fluctuations using the following three coupled dynamic equations:

$$
\begin{aligned}
& \langle\delta \dot{b}\rangle=-\mathrm{i} g_{0}\left(c_{2 s}\left\langle\delta c_{2}^{\dagger}\right\rangle-c_{1 s}^{*}\left\langle\delta c_{1}\right\rangle\right)-\frac{\gamma_{m}}{2}\langle\delta b\rangle, \\
& \left\langle\delta \dot{c}_{1}\right\rangle=-\kappa\left\langle\delta c_{1}\right\rangle+\mathrm{i} g_{0} c_{1 s}\langle\delta b\rangle+\varepsilon_{p} \mathrm{e}^{-\mathrm{i} x t} \\
& \left\langle\delta \dot{c}_{2}\right\rangle=-\kappa\left\langle\delta c_{2}\right\rangle-\mathrm{i} g_{0} c_{2 s}\left\langle\delta b^{\dagger}\right\rangle .
\end{aligned}
$$

We assume that the steady-state solutions of the above equations have the form $\langle\delta s\rangle=\delta s_{+} \mathrm{e}^{-\mathrm{i} x t}+\delta s_{-} \mathrm{e}^{\mathrm{i} x t}$, where $s=b, c_{1}, c_{2}$. Then it is straightforward to obtain

$$
\begin{aligned}
\delta b_{+} & =\frac{\mathrm{i} G \varepsilon_{p}}{(\kappa-\mathrm{i} x)\left(\frac{\gamma_{m}}{2}-\mathrm{i} x\right)+G^{2}\left(1-n^{2}\right)}, \\
\delta c_{1+} & =\frac{\varepsilon_{p}\left[-n^{2} G^{2}+(\kappa-\mathrm{i} x)\left(\frac{\gamma_{m}}{2}-\mathrm{i} x\right)\right]}{(\kappa-\mathrm{i} x)^{2}\left(\frac{\gamma_{m}}{2}-\mathrm{i} x\right)+G^{2}\left(1-n^{2}\right)(\kappa-\mathrm{i} x)}, \\
\delta c_{2-} & =\frac{-n G^{2} \varepsilon_{p}}{(\kappa+\mathrm{i} x)^{2}\left(\frac{\gamma_{m}}{2}+\mathrm{i} x\right)+G^{2}\left(1-n^{2}\right)(\kappa+\mathrm{i} x)},
\end{aligned}
$$

where $G=g_{0} c_{1 s}$ is the effective optomechanical coupling rate, and $\left|c_{2 s} / c_{1 s}\right|^{2}=n^{2}$ is the photon number ratio of two cavity modes. In deriving Eq. (7), we also assumed that $c_{1 s, 2 s}$ is real-valued without loss of generality.

On the basis of Eq. (7), we can further determine the left-hand output field $\varepsilon_{\text {out } L}$ and the right-hand output field $\varepsilon_{\text {out } R}$ using the following input-output relation [46]:

$$
\begin{aligned}
& \varepsilon_{\text {out } L}=2 \kappa\left\langle\delta c_{1}\right\rangle-\varepsilon_{p} \mathrm{e}^{-\mathrm{i} x t}, \\
& \varepsilon_{\text {out } R}=2 \kappa\left\langle\delta c_{2}\right\rangle,
\end{aligned}
$$

where the oscillating terms can be removed if we set $\varepsilon_{\text {out } L}=\varepsilon_{\text {out } L+} \mathrm{e}^{-\mathrm{i} x t}+\varepsilon_{\text {out } L-} \mathrm{e}^{\mathrm{i} x t}$ and $\varepsilon_{\text {out } R}=$ $\varepsilon_{\text {out } R+} \mathrm{e}^{-\mathrm{i} x t}+\varepsilon_{\text {out } R-} \mathrm{e}^{\mathrm{i} x t}$. Note that the output components $\varepsilon_{\text {out } L+}$ and $\varepsilon_{\text {out } R-}$ have the same frequency $\omega_{p}$ as the input probe fields $\varepsilon_{p}$, whereas the output components $\varepsilon_{\text {out } L-}$ and $\varepsilon_{\text {out } R+}$ are generated at frequencies of $2 \omega_{c}-\omega_{p}$ and $2 \omega_{d}-\omega_{p}$, respectively, in a nonlinear wave-mixing process of optomechanical interaction. Then, from Eq. (8) we can obtain

$$
\begin{aligned}
& \varepsilon_{\text {out } L_{+}}=2 \kappa \delta c_{1+}-\varepsilon_{p}, \\
& \varepsilon_{\text {out } R_{-}}=2 \kappa \delta c_{2-}
\end{aligned}
$$

with oscillation at a frequency $\omega_{p}$ of special interest.

In this paper, we discuss the perfect optomechanically induced amplification and transparency under realistic parameters in an optomechanical experiment [25]. That is, $L=25 \mathrm{~mm}, m=145 \mathrm{ng}, \kappa=2 \pi \times 215 \mathrm{kHz}$, $\omega_{m}=2 \pi \times 947 \mathrm{kHz}$, and $\gamma_{m}=2 \pi \times 141 \mathrm{~Hz}$. In addition, the laser wavelength is $\lambda=2 \pi c / \omega_{c}=1064 \mathrm{~nm}$, and the mechanical quality factor is $Q=\omega_{m} / \gamma_{m}=6700$.

\section{Perfect optomechanically induced transparency}

Here we consider perfect optomechanically induced transparency for the probe field. The quadrature of the optical components with frequency $\omega_{p}$ in the output field can be defined as $\varepsilon_{T}=2 \kappa \delta c_{1+} / \varepsilon_{p}$ [8]. Specifically, it can be written as

$$
\varepsilon_{T}=\frac{2 \kappa\left[-n^{2} G^{2}+(\kappa-\mathrm{i} x)\left(\frac{\gamma_{m}}{2}-\mathrm{i} x\right)\right]}{(\kappa-\mathrm{i} x)^{2}\left(\frac{\gamma_{m}}{2}-\mathrm{i} x\right)+G^{2}\left(1-n^{2}\right)(\kappa-\mathrm{i} x)},
$$

whose real and imaginary parts $\operatorname{Re}\left[\varepsilon_{T}\right]$ and $\operatorname{Im}\left[\varepsilon_{T}\right]$ represent the absorptive and dispersive behavior of the optomechanical system, respectively. It is well known that in a standard optomechanical system with a single optical cavity, the optomechanically induced transparency dip is not perfect, as the decay $\gamma_{m}$ of the MR is not zero. However, we can see from Eq. (10) that, in the doublecavity optomechanical system studied here, if the ratio $n=\sqrt{\gamma_{m} \kappa /\left(2 G^{2}\right)}$, the optomechanically induced transparency dip will be perfect, although a remarkable mechanical decay $\gamma_{m}$ exists.

To see this clearly, in Fig. 2, we plot $\operatorname{Re}\left[\varepsilon_{T}\right]$ versus the normalized frequency $x / \kappa$ with $\gamma_{m}=2 \pi \times 14.1 \mathrm{kHz}$ 


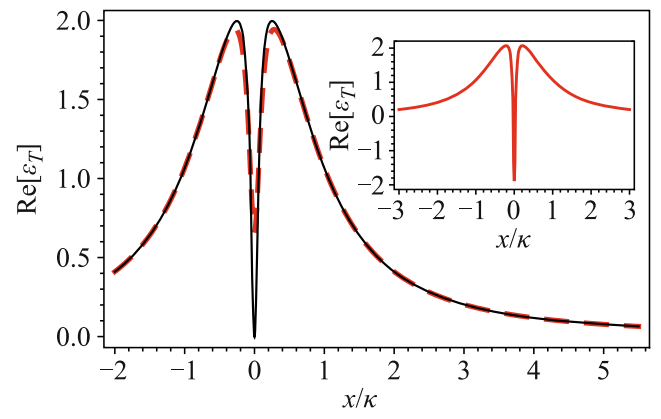

Fig. 2 The real part of $\varepsilon_{T}$ vs. the normalized frequency detuning $x / \kappa: n=0$ (red dashed line) and $n=0.7$ (black solid line) with $\gamma_{m}=2 \pi \times 14.1 \mathrm{kHz}$ and $\wp_{c}=1 \mathrm{~mW}$. In the inset: $n=0.7$, $\gamma_{m}=2 \pi \times 141 \mathrm{~Hz}$ and $\wp_{c}=1 \mathrm{~mW}$.

and $\wp_{c}=1 \mathrm{~mW}$ for different $n$. When $n=0$ (i.e., the usual optomechanically induced transparency case), the optomechanically induced transparency dip will become shallow with a large mechanical decay $\gamma_{m}$ (red dashed line). However, when an additional blue-sideband driving field satisfying the condition $n=\sqrt{\gamma_{m} \kappa /\left(2 G^{2}\right)} \approx 0.7$ is applied, the transparency dip will become perfect, exhibiting total transmission of the probe laser (black solid line). Physically, this means that the energy dissipated by the decay $\gamma_{m}$ of the MR can be compensated by applying a right-hand driving field with amplitude $\varepsilon_{d}=\varepsilon_{c} \sqrt{\gamma_{m} \kappa /\left(2 G^{2}\right)}$ and the mechanical blue sideband frequency. When $\omega_{p} \approx \omega_{0}, n=\sqrt{\gamma_{m} \kappa /\left(2 G^{2}\right)}$ and the beat frequency $\omega_{p}-\omega_{c}=\omega_{m}(x=0)$; thus, the MR is driven by a force oscillating at its eigenfrequency $\omega_{m}$, and the resonator starts to oscillate coherently. This motion will generate photons of frequency $\omega_{p}$ that interfere destructively with the probe beam, leading to an optomechanically induced transparency dip.

In Fig. 3, we plot the dispersion curve $\operatorname{Im}\left[\varepsilon_{T}\right]$ versus the normalized frequency $x / \kappa$ with $\gamma_{m}=2 \pi \times 14.1 \mathrm{kHz}$ and $\wp_{c}=1 \mathrm{~mW}$ for different $n$. Clearly, the curve with $n=0.7$ (black solid line) is much steeper than that with $n=0$ (red dashed line) near $x=0$. This means that we can easily control the dispersive behavior of the optomechanical system by applying a blue-detuned driving field

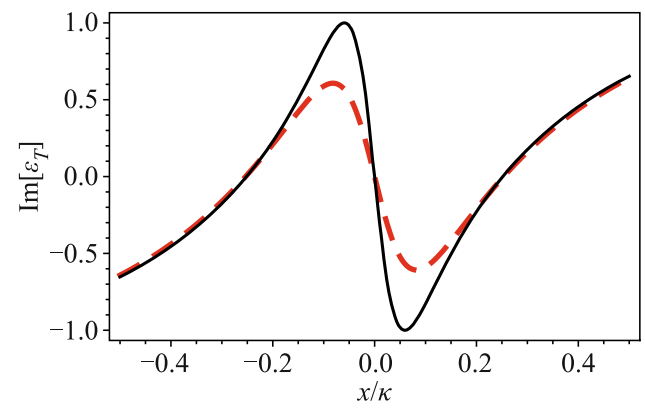

Fig. 3 The imaginary part of $\varepsilon_{T}$ vs. the normalized frequency detuning $x / \kappa: n=0$ (red dashed line) and $n=0.7$ (black solid line) with $\gamma_{m}=2 \pi \times 14.1 \mathrm{kHz}$ and $\wp_{c}=1 \mathrm{~mW}$. with amplitude $\varepsilon_{d}=n \varepsilon_{c}$, which can possibly be used to control slow light in optomechanical systems.

\section{Optomechanically induced amplification}

In this section, we study optomechanically induced amplification in this double-cavity optomechanical system. If the ratio $n>\sqrt{\gamma_{m} \kappa /\left(2 G^{2}\right)}$, we find that $\operatorname{Re}\left[\varepsilon_{T}\right]$ will become negative near $x=0$ (see the inset in Fig. 2). This means that an optomechanically induced gain (amplification) can be realized in this double-cavity system by applying a blue-detuned driving field to the right-side cavity with amplitude $\varepsilon_{d}=n \varepsilon_{c}$. Note that when the system operates under the condition $x=0$ and $n=\sqrt{1+\frac{\gamma m \kappa}{2 G^{2}}}$, $\operatorname{Re}\left[\varepsilon_{T}\right]$ will be divergent. In addition, the system will move into the parametric instability regime as $n \gtrsim 1$ when the input power $\wp_{c}=1 \mathrm{~mW}$, so we limit ourselves to the case where $n \leqslant 1$.

In Fig. 4, we plot the mechanical oscillation $\left|\kappa \delta b_{+} / \varepsilon_{p}\right|^{2}$ (normalized to the probe field $\varepsilon_{p}$ ) versus the normalized frequency $x / \kappa$ for different $n$. In the inset, we plot $\left|\kappa \delta b_{+} / \varepsilon_{p}\right|^{2}$ as a function of $n$ for $x=0$. The mechanical oscillation peak value is clearly located at $x=0$ and increases with increasing $n[n=0$ (black dotted line), $n=0.7$ (green dot-dashed line), $n=0.8$ (blue dashed line), $n=0.9$ (red solid line)]. Further, when $n$ increases to 1 , the mechanical oscillation peak value will increase to approximately $6.1 \times 10^{5}$ (see the inset in Fig. 4). This means that the optomechanical effect will become stronger for larger $n$ (less than or equal to 1 ) when $\omega_{p}-\omega_{c}=\omega_{m}(x=0)$ and $\omega_{d}-\omega_{0}=\omega_{m}$. The reason for this is that the mechanical blue sideband (heating sideband) of the right-hand cavity generates many phonons that will be absorbed by anti-Stokes processes in the left-hand cavity for the mechanical red sideband (cooling sideband). Thus, the optomechanical effect of the double-cavity system is resonantly enhanced.

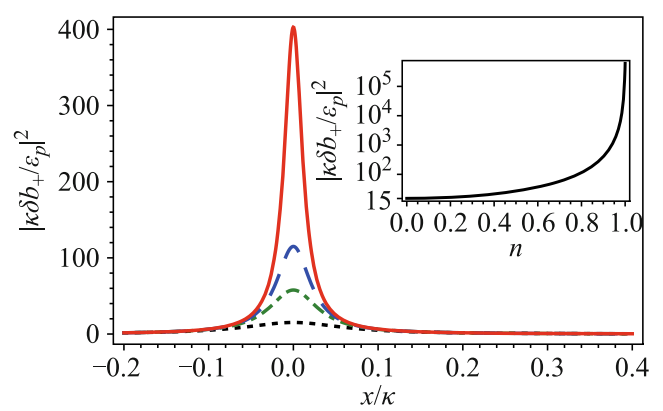

Fig. 4 The normalized mechanical oscillation $\left.\left|\kappa \delta b_{+}\right| \varepsilon_{p}\right|^{2}$ vs. the normalized frequency detuning $x / \kappa: n=0$ (black dotted line), $n=0.7$ (green dot-dashed line), $n=0.8$ (blue dashed line), and $n=0.9$ (red solid line) with $\wp_{c L}=1 \mathrm{~mW}$. In the inset, we plot the normalized mechanical oscillation $\left|\kappa \delta b_{+} / \varepsilon_{p}\right|^{2}$ vs. the ratio $n$. 
In Figs. 5 and 6 , we plot the output power $\left|\varepsilon_{\text {out } L+} / \varepsilon_{p}\right|^{2}$ and $\left.\left|\varepsilon_{\text {out } R-}\right| \varepsilon_{p}\right|^{2}$ normalized to the input probe field $\varepsilon_{p}$, respectively, versus the normalized frequency $x / \kappa$ for different $n$. The output energies $\left|\varepsilon_{\text {out } L+} / \varepsilon_{p}\right|^{2}$ and $\left.\left|\varepsilon_{\text {out } R-}\right| \varepsilon_{p}\right|^{2}$ clearly reach their maximum values at $x=0$ for a certain value of $n$. When $x=0$, the output normalized energies $\left|\varepsilon_{\text {out } L+} / \varepsilon_{p}\right|^{2}$ and $\left|\varepsilon_{\text {out } R-} / \varepsilon_{p}\right|^{2}$ will increase with $n$, which is similar to the mechanical oscillation $\left|\kappa \delta b_{+} / \varepsilon_{p}\right|^{2}$. This is because when $x=0$, the optomechanical effect will be strongest for a certain value of $n$, as discussed above. The curves of the output normalized energies $\left|\varepsilon_{\text {out } L+} / \varepsilon_{p}\right|^{2}$ and $\left|\varepsilon_{\text {out } R-} / \varepsilon_{p}\right|^{2}$ have almost the same line shape, except that the output normalized energy $\left|\varepsilon_{\text {out L }+} / \varepsilon_{p}\right|^{2}$ starts from 1 with increasing $n$ for $x=0$, whereas the output normalized energy $\left|\varepsilon_{\text {out } R-} / \varepsilon_{p}\right|^{2}$ starts from 0 (see the insets in Figs. 5 and 6 ). This shows that the double-cavity optomechanical system will be reduced to the standard one-cavity optomechanical model $\left(\left|\varepsilon_{\text {out } R-} / \varepsilon_{p}\right|^{2}=0\right)$ when $n=0$. When $n$ increases to 1 , the output normalized energies $\left|\varepsilon_{\text {out } L+} / \varepsilon_{p}\right|^{2}$ and $\left|\varepsilon_{\text {out } R-} / \varepsilon_{p}\right|^{2}$ will increase to approximately $1.6 \times 10^{5}$ (see the insets in Figs. 5 and 6 ). The reason for this is that the presence of the blue-detuned driving field with $\omega_{d}-\omega_{0}=\omega_{m}$ will coherently enhance

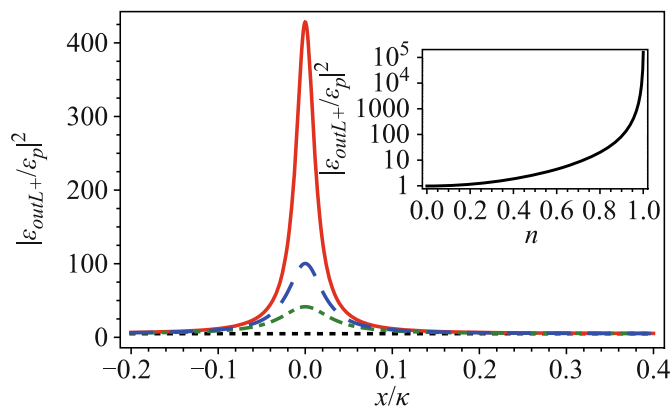

Fig. 5 The normalized left-hand output energy $\left.\left|\varepsilon_{\text {out } L+}\right| \varepsilon_{p}\right|^{2}$ vs. the normalized frequency detuning $x / \kappa: n=0$ (black dotted line), $n=0.7$ (green dot-dashed line), $n=0.8$ (blue dashed line), and $n=0.9$ (red solid line) with $\wp_{c L}=1 \mathrm{~mW}$. In the inset, we plot the normalized output energy $\left.\left|\varepsilon_{\text {out } L+}\right| \varepsilon_{p}\right|^{2}$ vs. the ratio $n$.

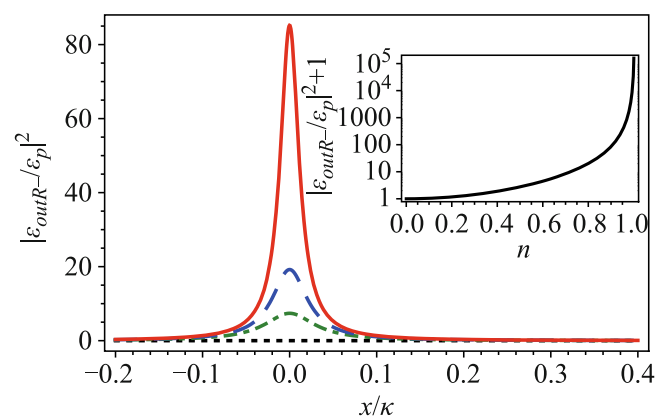

Fig. 6 The normalized right-hand output energy $\left|\varepsilon_{\text {out } R-} / \varepsilon_{p}\right|^{2}$ vs the normalized frequency detuning $x / \kappa$; the parameters are the same as in Fig. 4. In the inset, we plot the normalized output energy $\left|\varepsilon_{\text {out } R-}-\varepsilon_{p}\right|^{2}+1$ vs. the ratio $n$. the oscillation of the MR (see Fig. 4), leading to optomechanically induced amplification. Thus, we can realize optomechanically induced amplification for a resonantly injected probe in the double-cavity optomechanical system by appropriately adjusting the ratio $n$ of the two strong field amplitudes $\varepsilon_{c, d}$.

\section{Conclusions}

In summary, we theoretically studied a double-cavity optomechanical system driven by a red sideband laser from one side and a blue sideband laser from the other side. Our analytical and numerical results show that if the amplitude ratio of the two driving fields $n$ is adjusted so that $n>\sqrt{\gamma_{m} \kappa /\left(2 G^{2}\right)}$, optomechanically induced amplification of a resonantly incident probe (i.e., $\omega_{p}-\omega_{c}-\omega_{m}=0$ ) can be realized in this system. Typically, remarkable amplification can be obtained when $n \sim 1$. The reason for this is as follows: The Stokes processes in the blue-sideband-driven cavity can generate phonons in the mechanical elements, and these phonons will be further absorbed by anti-Stokes processes in the red-sidebanddriven cavity. As a result, the optomechanical effect of the double-cavity system is resonantly enhanced. In addition, perfect optomechanically induced transparency can be realized if we set $n=\sqrt{\gamma_{m} \kappa /\left(2 G^{2}\right)}$. Unlike the usual optomechanically induced transparency, this phenomenon is robust to mechanical dissipation; namely, the perfect transparency window can be preserved even if the MR has a relatively large decay rate $\gamma_{m}$. Our results indicate important progress toward optical signal amplification, light storage, fast light, and slow light in quantum information processes.

Acknowledgements This work was supported by the National Natural Science Foundation of China (Grant Nos. 61378094 and 11174027) and the Natural Science Foundation of Heilongjiang Province, China (No. A201402). W. Z. Jia was supported by the National Natural Science Foundation of China under Grants Nos. 11347001 and 11404269, the Fundamental Research Funds for the Central Universities (Grant No. 2682014RC21).

Open Access This article is distributed under the terms of the Creative Commons Attribution License which permits any use, distribution, and reproduction in any medium, provided the original author(s) and the source are credited.

\section{References}

1. T. J. Kippenberg and K. J. Vahala, Cavity optomechanics: Back-action at the mesoscale, Science 321(5893), 1172 (2008)

2. F. Marquardt and S. M. Girvin, Optomechanics, Physics 2, 
40 (2009)

3. P. Verlot, A. Tavernarakis, T. Briant, P. F. Cohadon, and A. Heidmann, Back-action amplification and quantum limits in optomechanical measurements, Phys. Rev. Lett. 104(13), $133602(2010)$

4. S. Mahajan, T. Kumar, A. B. Bhattacherjee, and ManMohan, Ground-state cooling of a mechanical oscillator and detection of a weak force using a Bose-Einstein condensate, Phys. Rev. A 87(1), 013621 (2013)

5. Y. W. Hu, Y. F. Xiao, Y. C. Liu, and Q. H. Gong, Optomechanical sensing with on-chip microcavities, Front. Phys. 8(5), 475 (2013)

6. S. Gigan, H. Böhm, M. Paternostro, F. Blaser, G. Langer, J. Hertzberg, K. Schwab, D. Bäuerle, M. Aspelmeyer, and A. Zeilinger, Self-cooling of a micromirror by radiation pressure, Nature 444(7115), 67 (2006)

7. D. Kleckner and D. Bouwmeester, Sub-kelvin optical cooling of a micromechanical resonator, Nature 444(7115), 75 (2006)

8. G. S. Agarwal and Sumei Huang, Electromagnetically induced transparency in mechanical effects of light, Phys. Rev. A 81, 041803(R) (2010)

9. T. J. Kippenberg and K. J. Vahala, Cavity opto-mechanics, Opt. Express 15(25), 17172 (2007)

10. D. K. Armani, T. J. Kippenberg, S. M. Spillane, and K. J. Vahala, Ultra-high-Q toroid microcavity on a chip, Nature 421(6926), 925 (2003)

11. A. Schliesser, R. Rivière, G. Anetsberger, O. Arcizet, and T. J. Kippenberg, Resolved-sideband cooling of a micromechanical oscillator, Nat. Phys. 4(5), 415 (2008)

12. M. Eichenfield, J. Chan, R. M. Camacho, K. J. Vahala, and O. Painter, Optomechanical crystals, Nature 462(7269), 78 (2009)

13. Y. Li, J. Zheng, J. Gao, J. Shu, M. S. Aras, and C. W. Wong, Design of dispersive optomechanical coupling and cooling in ultrahigh-Q/V slot-type photonic crystal cavities, Opt. Express 18(23), 23844 (2010)

14. J. D. Thompson, B. M. Zwickl, A. M. Jayich, F. Marquardt, S. M. Girvin, and J. G. E. Harris, Strong dispersive coupling of a high-finesse cavity to a micromechanical membrane, $\mathrm{Na}$ ture 452(7183), 72 (2008)

15. H. K. Cheung, and C. K. Law, Nonadiabatic optomechanical Hamiltonian of a moving dielectric membrane in a cavity, Phys. Rev. A 84(2), 023812 (2011)

16. F. Brennecke, S. Ritter, T. Donner, and T. Esslinger, Cavity optomechanics with a Bose-Einstein condensate, Science 322(5899), 235 (2008)

17. K. Zhang, P. Meystre, and W. Zhang, Role reversal in a Bose-Condensed optomechanical system, Phys. Rev. Lett. 108(24), 240405 (2012)

18. K. Y. Zhang, L. Zhou, G. J. Dong, and W. P. Zhang, Cavity optomechanics with cold atomic gas, Front. Phys. 6(3), 237 (2011)
19. C. A. Regal, J. D. Teufel, and K. W. Lehnert, Measuring nanomechanical motion with a microwave cavity interferometer, Nat. Phys. 4(7), 555 (2008)

20. Z. L. Xiang, S. Ashhab, J. Q. You, and F. Nori, Hybrid quantum circuits: Superconducting circuits interacting with other quantum systems, Rev. Mod. Phys. 85(2), 623 (2013)

21. I. Wilson-Rae, N. Nooshi, W. Zwerger, and T. J. Kippenberg, Theory of ground state cooling of a mechanical oscillator using dynamical backaction, Phys. Rev. Lett. 99(9), 093901 (2007)

22. F. Marquardt, J. P. Chen, A. A. Clerk, and S. M. Girvin, Quantum theory of cavity-assisted sideband cooling of mechanical motion, Phys. Rev. Lett. 99(9), 093902 (2007)

23. Y. Li, L. A. Wu, and Z. D. Wang, Fast ground-state cooling of mechanical resonators with time-dependent optical cavities, Phys. Rev. A 83(4), 043804 (2011)

24. J. M. Dobrindt, I. Wilson-Rae, and T. J. Kippenberg, Parametric normal-mode splitting in cavity optomechanics, Phys. Rev. Lett. 101(26), 263602 (2008)

25. S. Gröblacher, K. Hammerer, M. Vanner, and M. Aspelmeyer, Observation of strong coupling between a micromechanical resonator and an optical cavity field, Nature 460(7256), 724 (2009)

26. J. D. Teufel, D. Li, M. S. Allman, K. Cicak, A. J. Sirois, J. D. Whittaker, and R. W. Simmonds, Circuit cavity electromechanics in the strong-coupling regime, Nature 471(7337), 204 (2011)

27. A. Kronwald and F. Marquardt, Optomechanically induced transparency in the nonlinear quantum regime, Phys. Rev. Lett. 111(13), 133601 (2013)

28. S. Weis, R. Rivière, S. Deléglise, E. Gavartin, O. Arcizet, A. Schliesser, and T. J. Kippenberg, Optomechanically Induced Transparency, Science 330(6010), 1520 (2010)

29. A. H. Safavi-Naeini, T. P. M. Alegre, J. Chan, M. Eichenfield, M. Winger, Q. Lin, J. T. Hill, D. E. Chang, and O. Painter, Electromagnetically induced transparency and slow light with optomechanics, Nature 472(7341), 69 (2011)

30. M. Karuza, C. Biancofiore, M. Bawaj, C. Molinelli, M. Galassi, R. Natali, P. Tombesi, G. Di Giuseppe, and D. Vitali, Optomechanically induced transparency in a membrane-in-the-middle setup at room temperature, Phys. Rev. A 88(1), 013804 (2013)

31. D. E. Chang, A. H. Safavi-Naeini, M. Hafezi, and O. Painter, Slowing and stopping light using an optomechanical crystal array, New J. Phys. 13(2), 023003 (2011)

32. V. Fiore, Y. Yang, M. C. Kuzyk, R. Barbour, L. Tian, and H. Wang, Storing optical information as a mechanical excitation in a silica optomechanical resonator, Phys. Rev. Lett. 107(13), 133601 (2011)

33. T. Kippenberg, H. Rokhsari, T. Carmon, A. Scherer, and K. Vahala, Analysis of radiation-pressure induced mechanical oscillation of an optical microcavity, Phys. Rev. Lett. 95(3), 033901 (2005) 
34. F. Marquardt, J. G. E. Harris, and S. M. Girvin, Dynamical multistability induced by radiation pressure in high-finesse micromechanical optical cavities, Phys. Rev. Lett. 96(10), 103901 (2006)

35. K. Vahala, M. Herrmann, S. Knünz, V. Batteiger, G. Saathoff, T. W. Hänsch, and T. Udem, A phonon laser, Nat. Phys. 5(9), 682 (2009)

36. F. Massel, T. T. Heikkilä, J. M. Pirkkalainen, S. U. Cho, H. Saloniemi, P. J. Hakonen, and M. A. Sillanpää, Microwave amplification with nanomechanical resonators, $\mathrm{Na}$ ture 480(7377), 351 (2011)

37. A. Nunnenkamp, V. Sudhir, A. K. Feofanov, A. Roulet, and T. J. Kippenberg, Quantum-limited amplification and parametric instability in the reversed dissipation regime of cavity optomechanics, arXiv: 1312.5867 (2013)

38. A. Metelmann and A. A. Clerk, Quantum-limited amplification via reservoir engineering, Phys. Rev. Lett. 112(13), 133904 (2014)

39. X. B. Yan, C. L. Cui, K. H. Gu, X. D. Tian, C. B. Fu, and J. H. Wu, Coherent perfect absorption, transmission, and synthesis in a double-cavity optomechanical system, Opt. Express 22(5), 4886 (2014)
40. M. Paternostro, D. Vitali, S. Gigan, M. S. Kim, C. Brukner, J. Eisert, and M. Aspelmeyer, Creating and probing multipartite macroscopic entanglement with light, Phys. Rev. Lett. 99(25), 250401 (2007)

41. M. Bhattacharya and P. Meystre, Trapping and cooling a mirror to its quantum mechanical ground state, Phys. Rev. Lett. 99(7), 073601 (2007)

42. Y. D. Wang, and A. A. Clerk, Using interference for high fidelity quantum state transfer in optomechanics, Phys. Rev. Lett. 108(15), 153603 (2012)

43. R. W. Andrews, R. W. Peterson, T. P. Purdy, K. Cicak, R. W. Simmonds, C. A. Regal, and K. W. Lehnert, Bidirectional and efficient conversion between microwave and optical light, Nat. Phys. 10(4), 321 (2014)

44. J. T. Hill, A. H. Safavi-Naeini, J. Chan, and O. Painter, Coherent optical wavelength conversion via cavity optomechanics, Nat. Commun. 3, 1196 (2012)

45. G. S. Agarwal and S. Huang, Nanomechanical inverse electromagnetically induced transparency and confinement of light in normal modes, New J. Phys. 16(3), 033023 (2014)

46. D. F. Walls and G. J. Milburn, Quantum Optics, Berlin: Springer-Verlag, 1994 